\title{
SEGUIMIENTO DE LA DINÁMICA POBLACIONAL DE Pseudococcus viburni (Signoret) (Hemiptera: Pseudococcidae) EN DOS TEMPORADAS EN UN HUERTO DE GRANADOS (Punica granatum L.) EN CHILE CENTRAL
}

\section{MONITORING OF POPULATION DYNAMICS OF Pseudococcus viburni (Signoret) (Hemiptera: Pseudococcidae) DURING TWO SEASONS IN A POMEGRANATE (Punica granatum L.) ORCHARD IN CENTRAL CHILE}

\author{
Carlos Carpio ${ }^{1}$, y Tomislav Curkovic ${ }^{2 *}$ \\ 1 Escuela Superior Politécnica de Chimborazo, Fac. de Recursos Naturales, Quito, Ecuador. fccarpio@ \\ yahoo.com \\ 2 Facultad de Ciencias Agronómicas, Depto. De Sanidad Vegetal, Universidad de Chile, Santiago, \\ Chile. \\ * Autor para correspondencia E-mail: tcurkovi@uchile.cl
}

\section{RESUMEN}

El chanchito blanco (Pseudococcus viburni) es la principal plaga del granado (Punica granatum $\mathrm{L}$.) en Chile, de manera que se requiere disponer de un sistema de seguimiento eficiente. Entre 2011 y 2013 se evaluaron cuatro métodos de seguimiento: cartón corrugado en troncos, cintas doble adhesivas en ramillas, revisión de frutos y revisión de follaje; el estudio se realizó en un huerto de granados, en la Región Metropolitana. El método más eficiente fue el cartón corrugado colocado alrededor de los troncos, a $120 \mathrm{~cm}$ de altura, que mostró migración de $P$. viburni desde los refugios invernales hacia el follaje desde septiembre, con capturas durante todo el año. Se evidenciaron tres generaciones (primavera, verano y otoño) anuales, aunque las poblaciones fueron bajas en el invierno. Hubo un incremento significativo de ninfas y hembras adultas en los cartones en abril-mayo (cosecha), atribuible a la emigración desde los frutos, que se parten naturalmente. En la revisión periódica de frutos se observó infestación en la cavidad calicinal desde el momento de la cuaja hasta la cosecha, encontrándose al momento de la cosecha más del $75 \%$ de los frutos infestados, a pesar del programa de insecticidas. El muestreo de follaje y de cintas doble adhesivas no fue efectivo para muestrear $P$. viburni. La infestación de las granadas no se correlacionó linealmente con los parámetros del fruto (peso o tamaño) ni con la densidad de pseudocóccidos en cartones, pero sí con el porcentaje de cartones infestados. Las variables climáticas no se correlacionaron significativamente con las densidades de chanchitos. Estos resultados servirán para definir mejor la oportunidad de aplicación de agroquímicos para el control de $P$. viburni en granados.

Palabras clave: cavidad calicinal, infestación de frutos, trampas de cartón corrugado.

\section{ABSTRACT}

Pseudococcus viburni is the main pomegranate pest in Chile, so an efficient monitoring system is required. Four monitoring methods were evaluated between 2011 and 2013 in a pomegranate orchard in the Metropolitan Region, Chile. The methods evaluated were: cardboards on trunks, doublesided tapes on branches, fruit sampling and foliage sampling. Cardboards placed on the trunk at $120 \mathrm{~cm}$ from the ground were the most efficient method, showing pseudococcid migration from overwintering sites to the foliage since the spring (September). Three annual generations (spring, 
summer, and autumn) were observed for $P$. viburni in cardboards, but low populations were found during the winter. There was an increasing number of nymphs and adult females under cardboards during April-May (harvest time), attributable to the emigration from naturally splitting fruits. An increasing fruit calycinal cavity infestation was observed from fruit set to harvest, reaching above $75 \%$ of sampled fruits even though an insecticide spray program was in place. Foliage sampling and double-sided tapes were not efficient for $P$. viburni monitoring. Fruit infestation was not linearly correlated either with fruit parameters (size or weight) or pseudococcid densities in cardboards, but it was correlated with the percentage of infested cardboards. Climatic variables did not exhibit any significant correlation with pseudococcid densities. These results will help improve the agrochemical spray timing against $P$. viburni in pomegranates.

Key words: calycinal cavity, cardboard traps, fruit infestation.

\section{INTRODUCCIÓN}

En los últimos años las exportaciones de granados (Punica granatum L.) se han incrementado a nivel mundial y por ello las plantaciones aumentaron notablemente en Chile; en 2010 había alrededor de 200 ha plantadas y en 2013 la superficie estimada de granados alcanzó las 885,1 ha (Henríquez, 2015), lo que se ha traducido en un aumento de los problemas fitosanitarios (Curkovic et al., 2015). Uno de los principales problemas de plagas del granado en nuestro país son los chanchitos blancos (Pseudococcus viburni), (Hemiptera: Pseudococcidae), que debido a su condición cuarentenaria limitan la comercialización de la fruta (Oyarzún, 2004). La detección de este tipo de insectos en las inspecciones de post-cosecha durante el proceso exportador ha provocado importantes rechazos de granadas frescas chilenas (Curkovic et al., 2015). Entre las especies de pseudocóccidos que afectan a los frutales en Chile, la principal es Pseudococcus viburni (Signoret) o chanchito blanco de la vid (Koplow, 2004), con varios hospederos frutales, incluyendo al granado (Ripa y Rodríguez, 1999) donde se considera la plaga más importante (Curkovic et al., 2015). Esta especie es ovípara, coloca sus huevos en ovisacos de aspecto algodonoso constituidos por fibras cerosas. Las hembras tienen tres estadios ninfales y son ápteras, mientras que los machos tienen cuatro estadios juveniles y los adultos son alados. La especie desarrolla tres generaciones por temporada en varios frutales estudiados en el país (Oyarzún, 2004), aunque en granados no hay antecedentes al respecto. Dados los hábitos crípticos de $P$. viburni es necesario un sistema de seguimiento eficiente que ayude a definir un control oportuno (Núñez, 2007). Existen algunos estudios sobre métodos de seguimiento de $P$. viburni en carozos (González et al., 1996), pomáceas (Mudavanhu et al., 2011) y vid (Koplow, 2004; Nuñez, 2007), pero no hay antecedentes en el granado. Debido a lo antes señalado, este estudio tuvo los siguientes objetivos: 1) comparar cuatro métodos de seguimiento del chanchito blanco en granados, y 2) estudiar la dinámica poblacional del chanchito blanco en el cultivo.

\section{MATERIALES Y MÉTODOS}

Huerto. El estudio se hizo durante las temporadas 2011-2012 y 2012-2013, en un huerto comercial de granados ubicado en Huechún $\left(33^{\circ} 4^{\prime} 58,44^{\prime \prime}\right.$ S, $70^{\circ} 40^{\prime} 10,45^{\prime \prime}$ O), TilTil, Región Metropolitana, Chile; se usaron plantas del cv. Wonderful plantadas a $3 \times 5 \mathrm{~m}$ en 2004. El programa fitosanitario contra $P$. viburni incluyó: $2,25 \mathrm{~kg} \mathrm{ha}^{-1}$ de diazinon $40 \mathrm{WP}$ (producto comercial asperjado con $2.200 \mathrm{~L} \mathrm{ha}^{-1}$ ) aplicado el 20 de septiembre de 2011; 1,5 L ha ${ }^{-1}$ de espirotetramato 100 SL (22 de octubre de 2011); $0,45 \mathrm{~kg} \mathrm{ha}^{-1}$ de imidacloprid $70 \mathrm{WP}$, aplicado el 12 y 28 de noviembre de 2011, y el 23 de febrero de 2012; $2,25 \mathrm{~kg} \mathrm{ha}^{-1}$ de diazinon 40 WP (5 de octubre de 2012); $1,2 \mathrm{~kg} \mathrm{ha}^{-1}$ de clorpirifos 75 WG (30 de octubre de 2012); y 0,45 $\mathrm{kg} \mathrm{ha}^{-1}$ de imidacloprid 70 WP, aplicado el 5 de diciembre y 26 de enero de 2012, y el 23 de febrero de 2013. El predio contaba con una estación meteorológica (PCE-FWS 20; PCE Instruments, Gran Bretaña) para medir la temperatura, precipitación y humedad relativa.

Métodos de seguimiento de $P$. viburni. Se seleccionaron sectores con infestación de $P$. viburni con un umbral de cuatro individuos por árbol, identificados después de realizar un muestreo preliminar en la plantación. Se evaluaron cuatro métodos de seguimiento: cartón corrugado, cinta doble adhesiva, revisión de frutos, y revisión de follaje. Se muestrearon 10 árboles/método (2 plantas/hilera, en 5 hileras alternadas) cada tres semanas, durante las temporadas 2011-2012 y 2012-2013. Se usaron diferentes árboles para cada método de muestreo. Se contó el número de individuos vivos de $P$. viburni, por estadio, con lupa estereoscópica (20x). 
Trampas de agregación. Se colocaron bandas de cartón corrugado (Cartonpack S.A., Quilicura, Santiago) de aproximadamente $10 \mathrm{~cm}$ de ancho, alrededor del tronco, a dos alturas en cada árbol: 30 y $120 \mathrm{~cm}$ del suelo. En cada fecha de muestreo se recolectaron los cartones antiguos y se remplazaron por nuevos. Se contaron los individuos presentes bajo el cartón y los que estaban entre las hojas de papel corrugado.

Trampas adhesivas. Se colocó una cinta adhesiva doble faz (Scotch Double Sided, 3M) alrededor de una rama con flores o frutos, en cinco ramas de cada árbol. En cada fecha se retiraron las cintas antiguas y se reemplazaron por nuevas.

Revisión de follaje y frutos. Se recolectó una rama de $20 \mathrm{~cm}$ y dos frutos por árbol; cuando no hubo frutos se recolectaron flores. En los frutos y flores se revisó la superficie y la cavidad calicinal, $\mathrm{y}$ en las ramas se revisaron todas las hojas. Adicionalmente, se midió el peso, diámetro y altura del fruto, y la profundidad de la cavidad calicinal.

Análisis de los resultados. Para contrastar los métodos de seguimiento, se utilizó un diseño de bloques completos al azar, con la fecha de muestreo como factor de bloqueo, en cada temporada, en el período de fructificación (diciembre-mayo). Se consideró la mediana de individuos registrados por árbol, debido a que las medias no tenían una distribución normal, incluso después de transformar los datos. Se hizo un análisis de conglomerados de variables y se aplicó la prueba no paramétrica de Friedman y la prueba de comparaciones múltiples de Conover (1999), con un nivel de confianza del $95 \%$. Se hicieron análisis de regresión lineal entre ambos, la mediana de los individuos móviles de $P$. viburni en cartones y el porcentaje de cartones ubicados a $120 \mathrm{~cm}$ infestados vs. el porcentaje de frutos infestados. Los datos de porcentaje se normalizaron por la transformación angular de Bliss (Steel y Torrie, 1985). Con los resultados de pseudocóccidos encontrados en los cartones corrugados y en los frutos se hicieron gráficas de la variación de la abundancia por estadio en el tiempo. También se hicieron análisis de regresión entre las variables climáticas, así como con las medidas tomadas en los frutos (peso y dimensiones del fruto; y profundidad de la cavidad calicinal), vs. la mediana de los individuos móviles de P. viburni durante 2012. Los datos de la mediana de los individuos móviles de $P$. viburni se transformaron con $\ln (x+0,5)$; mientras que los datos de precipitación y humedad relativa se normalizaron con $\log (x+0,5)$.

\section{RESULTADOS}

Comparación de métodos de seguimiento de P. viburni. El uso de trampas de agregación fue el método con significativamente mayores densidades de individuos móviles de $P$. viburni en ambas temporadas (Fig. 1), presentando los mejores resultados con los cartones colocados a $120 \mathrm{~cm}$ (cartón arriba). En las dos temporadas, los muestreos de frutos evidenciaron un resultado significativo pero intermedio, siempre inferior al "cartón arriba", mientras que en las trampas doble adhesivas y en las revisiones de follaje prácticamente no se encontraron individuos, siendo ambos estadísticamente similares, pero significativamente inferiores de los otros métodos.

Dinámica poblacional de $P$. viburni en granados. Todos los estadios de P. viburni fueron observados en los cartones a lo largo del año (temporada 2012-2013), aunque las poblaciones fueron bajas entre julio y septiembre. Se observó un patrón de fluctuación cíclica en todos los estados de desarrollo (Fig. 2), con tres máximos poblacionales a lo largo de la temporada. A inicios de la primavera se detectó la presencia de ninfas y luego de hembras en los cartones, lo que representaría el comienzo de la migración desde las raíces y otros refugios hacia el follaje. Las oviposturas, en general, comienzan desde fines de septiembre en adelante. Los machos se encuentran en densidades relativamente menores y en general coinciden con el máximo poblacional de las hembras, en septiembre. En ese período, no se observó presencia de chanchitos blancos en el interior de las flores. Se constató que el fruto (Fig. 3) es colonizado desde la cuaja y presenta infestación por ninfas, hembras adultas y ovisacos de $P$. viburni prácticamente durante toda la temporada, con máximos en febrero y mayo (inicio de la cosecha, por tanto no se pudo aplicar insecticidas) para hembras (4 individuos/fruto) y ovisacos ( $10 \%$ de frutos infestados), y durante marzo, para el caso de ninfas (6 individuos/ fruto). Se observó que en el interior del fruto del granado se completan dos generaciones (verano y otoño). A partir de junio se observó un elevado movimiento de ninfas desde las granadas hacia el resto de la planta. No se encontró una relación lineal significativa entre las variables climáticas y la mediana de la abundancia de $P$. viburni $(\mathrm{p}>$ $0,05)$ en el huerto de granados.

Infestación de frutos. En la Fig. 4 se observa la fluctuación de la mediana de los individuos en cartones y el nivel de infestación (\%) de los frutos a lo largo del período 2011-2013. Cabe señalar que se encontró hasta un $75 \%$ de los frutos infestados 

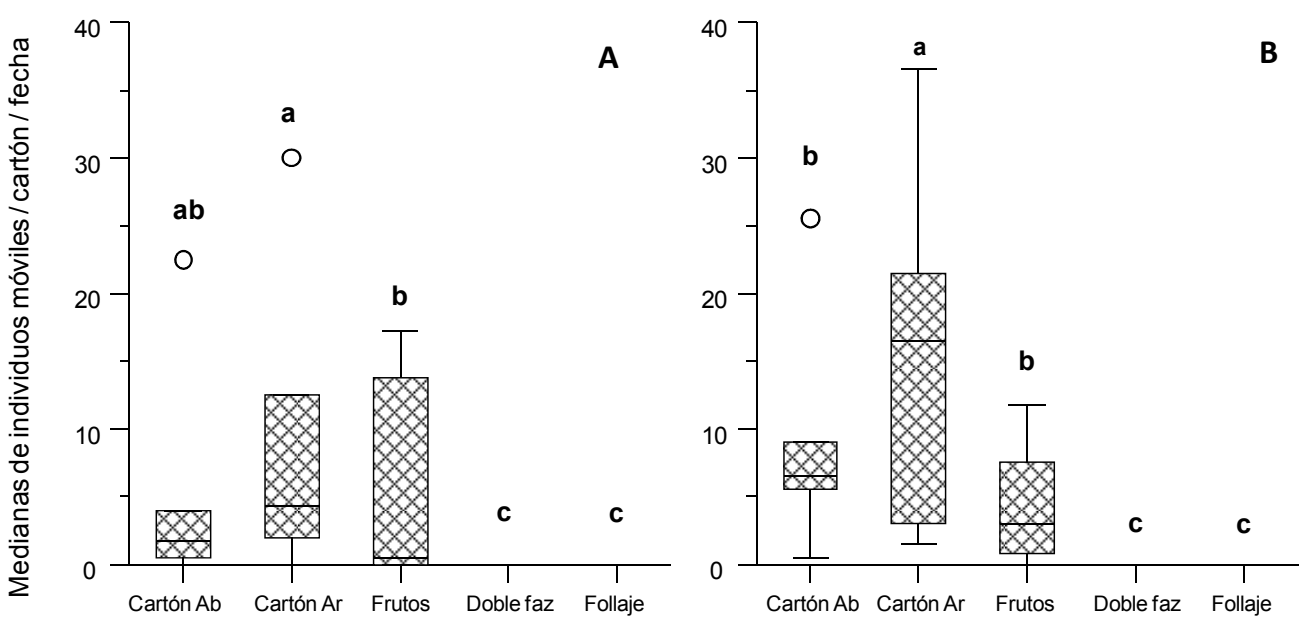

Fig. 1. Diagrama de caja de las medianas de individuos móviles de $P$. viburni/árbol/fecha en un campo de granado var. Wonderful (Huechún, Región Metropolitana, Chile), usando diferentes métodos de muestreo: cartones corrugados (abajo o "ab": a $30 \mathrm{~cm}$; arriba o "ar": a $120 \mathrm{~cm}$ del suelo); cintas adhesivas de doble faz; revisión de frutos y de hojas (follaje). Se muestran los resultados de las temporadas 2011-2012 (A) y 2012-2013 (B). En la caja está representada el 50\% de la muestra; el borde superior es el percentil 75 y el inferior es el percentil 25; la línea central es la mediana. Métodos de muestreo con diferente letra fueron estadísticamente diferentes según el test de Conover $(\mathrm{P} \leq 0,05)$.

Fig. 1. Box plot for the median of mobile individuals of $P$. viburni/tree/date found in a pomegranate orchard var. Wonderful (Huechún, Metropolitan Region, Chile), using different sampling methods: cardboards (ab: at $30 \mathrm{~cm}$; ar: at $120 \mathrm{~cm}$ above the ground); double-sided tapes; fruits and leaves. Data from both, the 2011-2012 (A) and 2012-2013 (B) seasons are shown. Within the box plot, $50 \%$ of the sample is presented; the upper box edge represents the 75 th percentile and the lower the 25th percentile; the central line represents the median. Sampling methods showing different letters were statistically different $(P \leq 0.05)$ after Conover test.

(al menos con un individuo vivo a cosecha) en la primera temporada y hasta $100 \%$ en la segunda, a pesar de un programa fitosanitario que incluyó cinco aplicaciones de insecticidas residuales cada año. De hecho, se encontró un promedio de 6 ninfas y hembras adultas en la cavidad calicinal de los frutos a cosecha (Carpio, 2013).

No se encontraron regresiones lineales significativas entre la mediana de la abundancia de los individuos móviles de P. viburni en cartones y el porcentaje de infestación de los frutos en ambas temporadas $(p>0,05)$. Sin embargo, en la Fig. 5 se observa la relación significativa que existe entre el porcentaje de cartones infestados (colocados a $120 \mathrm{~cm}$ del suelo) y el porcentaje de fruta infestada durante las dos temporadas, entre enero y mayo. En la primera temporada la relación pudo ser explicada con un modelo de regresión lineal con la ecuación: Frutos $(\%)=-$ $26,45+1,156 *$ (\% cartones), $\mathrm{F}=5,59 ; \mathrm{r}^{2}=0,57 ; \mathrm{p}$ $=0,049$; mientras que en la segunda temporada la relación se explicó mejor con un modelo de regresión cuadrática con la ecuación: Frutos
$(\%)=-957,4+27,33 *(\%$ cartones $)-0,1792$ * $(\% \text { cartones })^{2}, \mathrm{~F}=11,26 ; \mathrm{r}^{2}=0,81 ; \mathrm{p}=0,044$. Aunque las regresiones lineales fueron altamente significativas $(p<0,005)$, ninguno de las variables medidos en el fruto explican en alta proporción $\left(\mathrm{r}^{2}=0,021-0,20\right)$ la variación de la abundancia de individuos móviles de $P$. viburni encontrada al interior del fruto.

\section{DISCUSIÓN}

El uso de cartón corrugado ubicado alrededor del tronco a $120 \mathrm{~cm}$ del suelo resultó ser la metodología más eficaz para realizar el seguimiento de $P$. viburni en el huerto de granados. El hábito de crecimiento del granado que es arbustivo y con tendencia a ramificar desde la base (Frank et al., 2015), hace que las trampas ubicadas a $120 \mathrm{~cm}$ estén en el área de influencia de las aplicaciones foliares y que esto se vea reflejado en los conteos de los cartones corrugados. Este método mostró anteriormente su eficacia para el muestreo de estos insectos en 

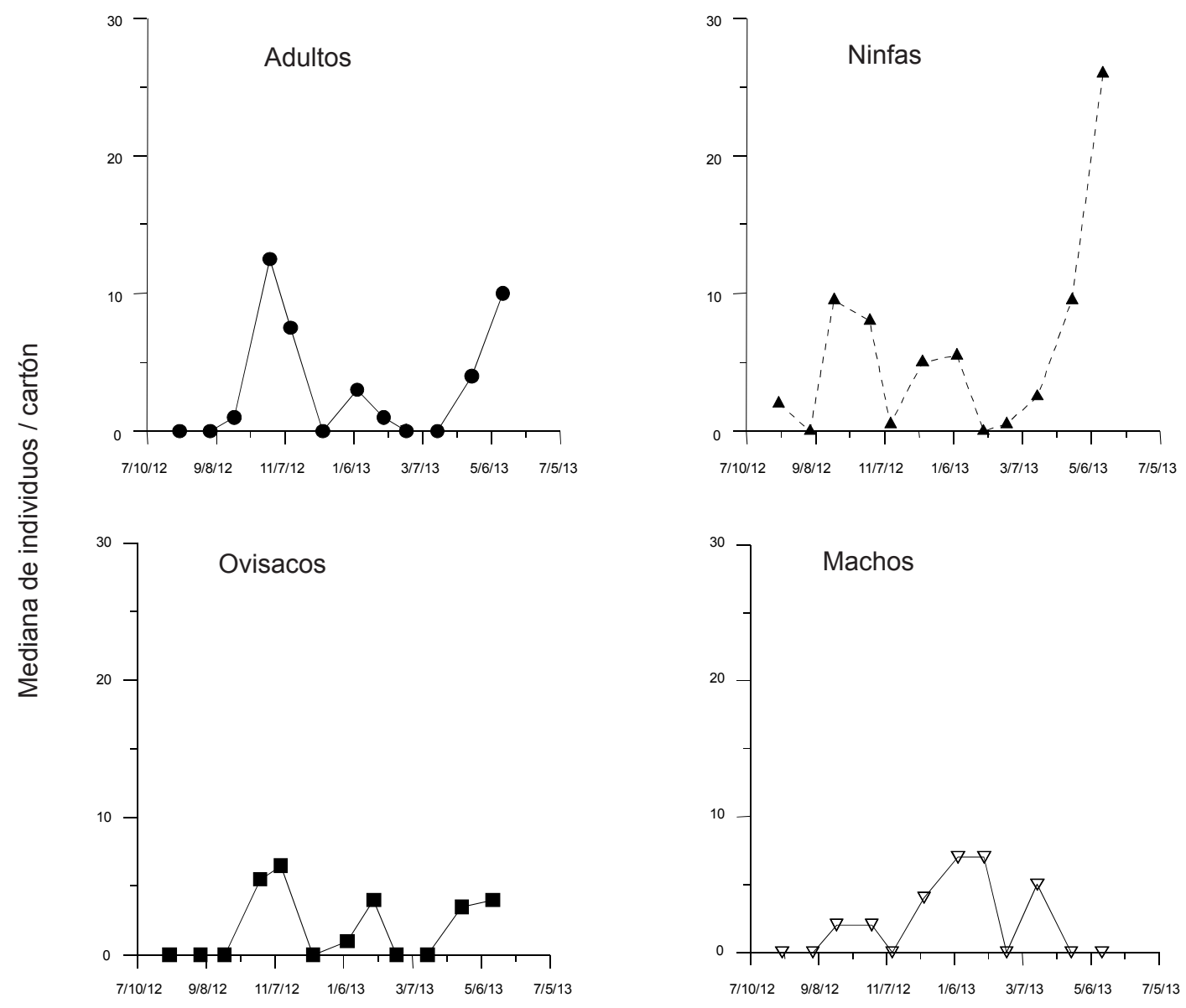

Fechas monitoreo

Fig. 2. Capturas de hembras adultas, ninfas, machos y ovisacos de P. viburni en cartones corrugados ubicados en troncos de granados a $120 \mathrm{~cm}$ del suelo, en varias fechas entre el 7 de agosto 2012 y el 16 de mayo de 2013 en una plantación de la variedad Wonderful, Huechún, Región Metropolitana, Chile.

Fig. 2. Captures of females, nymphs, males, and ovisacs of $P$. viburni in cardboards placed on pomegranate trunks at $120 \mathrm{~cm}$ above the ground, on several dates from August 7th, 2012 through May $16^{\text {th }}, 2013$, in an orchard of the variety Wonderful, Huechún, Metropolitan Region, Chile.

carozos (González et al., 1996), vides (Koplow, 2004; Nuñez, 2007), pomáceas (Mudavanhu, 2009) y olivos (Curkovic y Ballesteros, 2011). Koplow (2004) mencionó que el uso de cartón corrugado resultó ser sencillo y presentó altas correlaciones con el nivel de infestación de los frutos en vid a cosecha, similar a lo encontrado en el presente estudio. Vreysen (2005) menciona algunos aspectos que requiere un dispositivo de trampeo para un programa de seguimiento: correlación inversa entre el número de trampas y la eficiencia de trampeo; mínimo tiempo requerido para colocar y atender la trampa; bajo costo de cada unidad; alta especificidad de las capturas.
En el presente estudio, aunque los cartones no son específicos, con pocas unidades se pudo conocer el nivel de infestación y la dinámica poblacional de P. viburni en granado; además los materiales son de bajo costo y fáciles de conseguir, y se necesita poco tiempo para instalarlos y retirarlos (González et al., 1996).

Si bien los frutos del granado son estructuras que permiten la instalación de poblaciones de chanchitos en su cavidad calicinal (perianto del fruto), y su revisión periódica sirve para conocer la dinámica poblacional, y se complementa con la información obtenida con los cartones; el muestreo de frutos no puede ser utilizado como un sistema predictivo, debido a que cuando el 


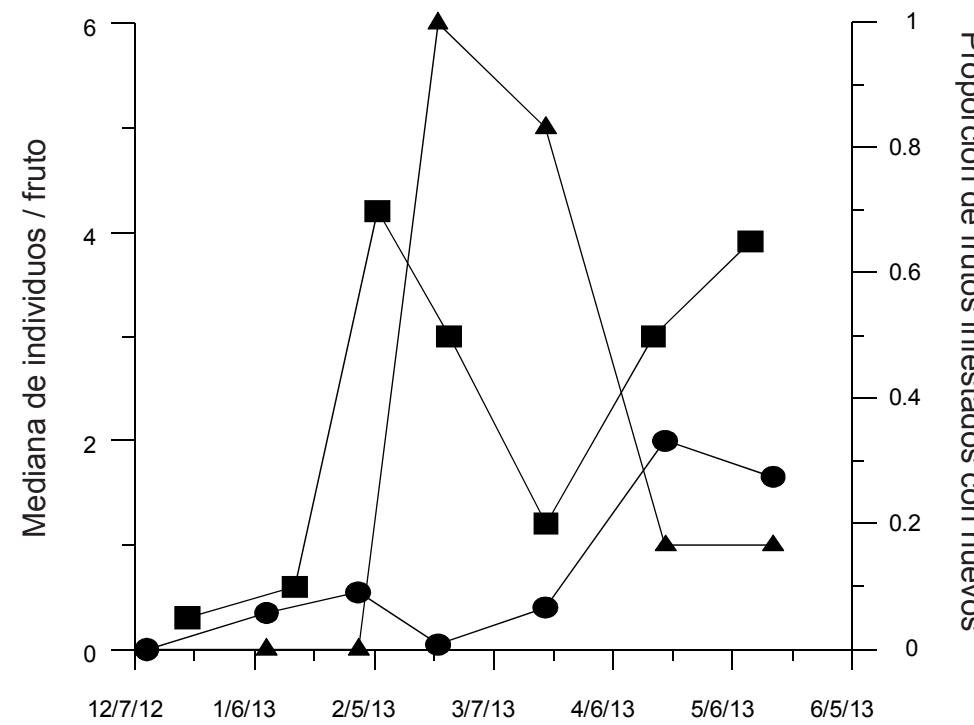

- Adultos

- Ninfas

Proporción frutos infestados

Fig. 3. Mediana de hembras adultas y ninfas de $P$. viburni por fruto, y proporción de frutos infestados con huevos en la cavidad calicinal, entre el 10 de diciembre 2012 y el 16 de mayo de 2013, en una plantación de granados var. Wonderful, Huechún, Región Metropolitana, Chile.

Fig. 3. Median of $P$. viburni adult females and nymphs per fruit, and proportion of calycinal cavities infested with egg sacs, between December $10^{\text {th }}, 2012$ through May $16^{\text {th }}, 2013$, in an orchard of pomegranate cv. Wonderful, Huechún, Metropolitan Region, Chile.

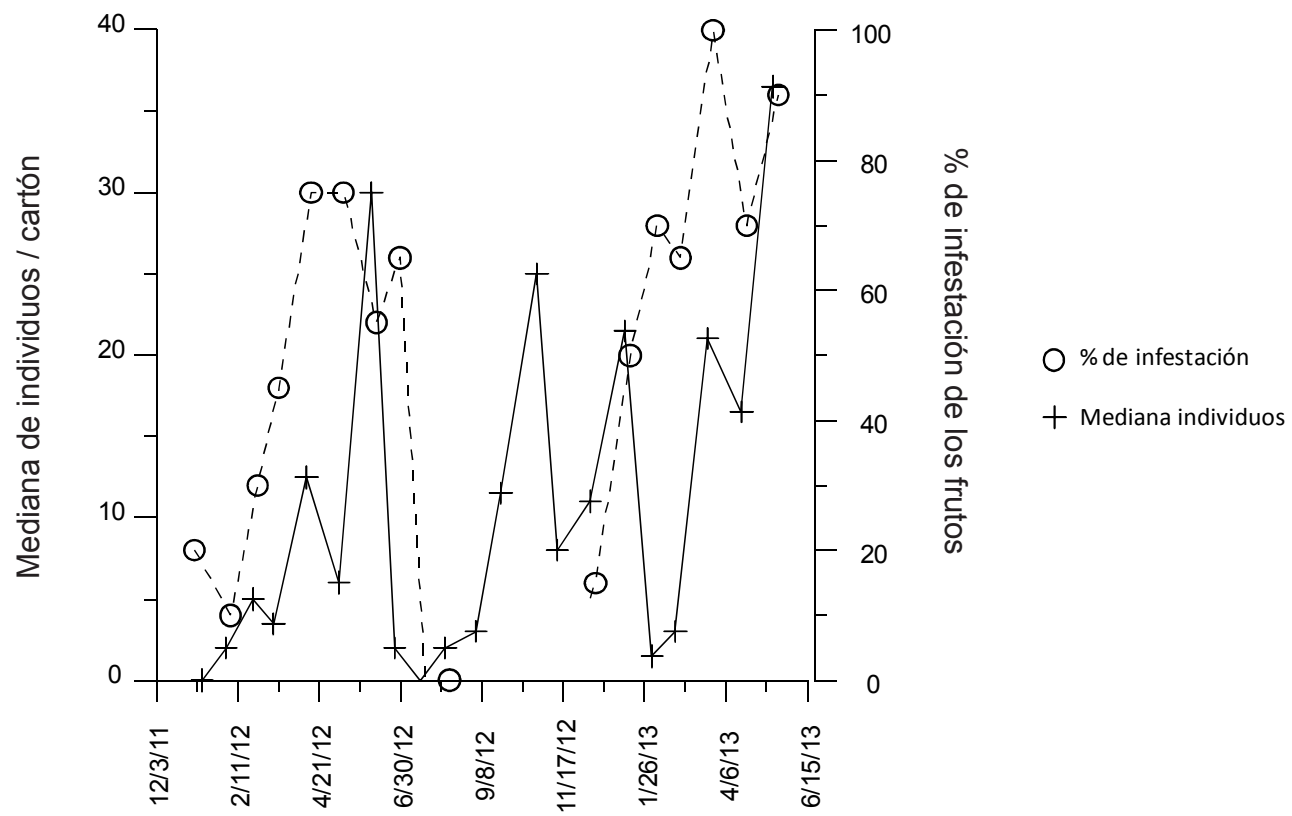

Fig. 4. Mediana de individuos móviles de $P$. viburni en cartones ubicados en troncos a $120 \mathrm{~cm}$ del suelo vs. porcentaje de infestación en frutos de granado var. Wonderful, durante dos temporadas (2011-2013), Huechún, Región Metropolitana, Chile.

Fig. 4. Median of mobile individuals of $P$. viburni in cardboards placed on trunks at $120 \mathrm{~cm}$ above the ground vs. infestation percentage in fruits of pomegranate $\mathrm{cv}$. Wonderful, during two seasons (2011-2013), Huechún, Metropolitan Region, Chile. 


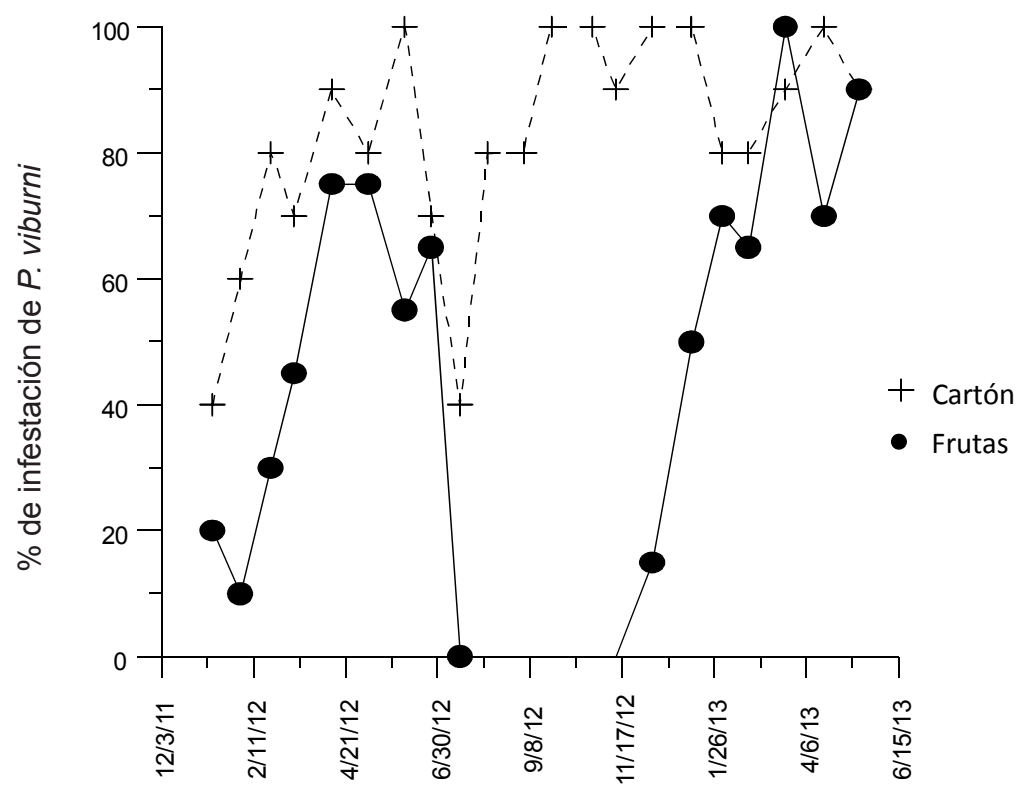

Fig. 5. Niveles de infestación (\%) con individuos móviles de $P$. viburni en frutos o cartones colocados en troncos a $120 \mathrm{~cm}$ del suelo, en granados var. Wonderful durante dos temporadas (20112013), Huechún, Región Metropolitana, Chile.

Fig. 5. Level of infestation (\%) with mobile individuals of $P$. viburni in fruits and cardboards placed on trunks at $120 \mathrm{~cm}$ above the ground, on pomegranates var. Wonderful, during two seasons (2011-2013), Huechún, Metropolitan Region, Chile.

insecto ha ingresado al fruto de granado es muy difícil eliminar. Cabe señalar que los niveles de infestación encontrados a cosecha fueron altos (75 a 100\% entre ambas temporadas), lo que representa un enorme riesgo de detección de estos insectos en las eventuales inspecciones fitosanitarias de post cosecha. Considerando que en el huerto se implementó un intenso programa fitosanitario contra pseudocóccidos, es posible que la selección de insecticidas y la oportunidad y método de aplicación no hayan sido adecuados para estos propósitos.

Se puede observar que después de la aplicación de los productos químicos (Fig. 6), hay una disminución de la población en los cartones, sin embargo el porcentaje de infestación observado en los frutos tiene un incremento sostenido desde el momento en que estos comienzan a formarse, y este parece ser levemente afectado por las aplicaciones, excepto en la última aplicación, cuando del $100 \%$ baja al $70 \%$ de infestación, aunque después de tres semanas vuelve a subir al $90 \%$. En general el control químico de estos insectos presenta dificultades que permiten parcialmente su sobrevivencia en frutales (González et al., 1996). Con respecto a los dos sistemas menos eficientes para el seguimiento del chanchito blanco en el granado, la revisión del follaje y el uso de cintas doble adhesivas, los resultados coinciden con lo planteado en la literatura para otros frutales (González et al., 1996; Oyarzún, 2004; Nuñez, 2007). Por último, la diferencia en poblaciones obtenidas en cartones entre ambas temporadas podría deberse a los distintos niveles de infestación inicial; un $40 \%$ de los cartones infestados a la cuaja en la temporada 2011-2012, mientras que en la temporada 20122013 esa cifra se duplicó a igual época.

La abundancia de $P$. viburni encontrada en los cartones no se pudo correlacionar con el nivel de infestación encontrado en los frutos, posiblemente debido a la estructura del fruto. En la cavidad calicinal, formada a partir del cáliz de la flor, el chanchito blanco encuentra un refugio seguro en donde tiene alimento, un micro-ambiente favorable, y protección contra enemigos naturales y contra la aplicación de insecticidas. Varias investigaciones, a través de modelos lineales, han podido explicar la relación entre el sistema de trampeo y la presencia de pseudocóccidos o el porcentaje de daño a cosecha (Koplow, 2004; Nuñez, 2007; Geiger and Daane, 2001; Mudavanhu et al., 2011; Waterworth et al., 2011). Sin embargo, en el presente estudio en la segunda temporada la relación del porcentaje de infestación (90\%) encontrado en los cartones 


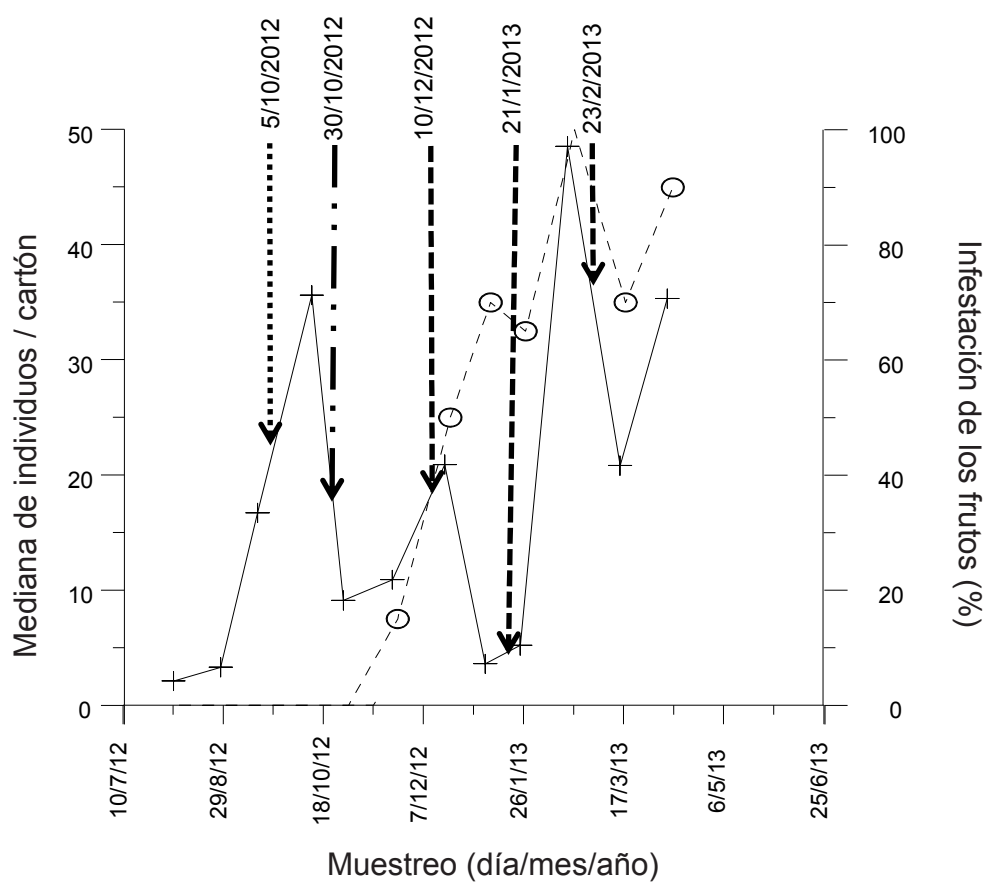

Fig. 6. Programa de insecticidas aplicados en el huerto de granados (Huechún, Región Metropolitana), en la temporada 2012-2013. Las flechas muestran las fechas de aplicación (diazinon: clorpirifos: $\ldots$. , e imidacloprid: $-\ldots+\ldots),++=$ representa la mediana de los individuos vivos de Pseudococcus viburni en cartones, $\mathrm{O}=$ es el porcentaje de infestación de frutos.

Fig. 6. Insecticide program applied on the pomegranate orchard (Huechún, Metropolitan Region) during the 2012-2013 season; arrows show application dates (diazinon: .........., chlorpyrifos: - .., and imidacloprid: $-\ldots \ldots$ ). $++=$ is the median of mobile individuals Pseudococcus viburni in cardboards at $120 \mathrm{~cm}$ above the ground, $\mathrm{O}=$ is the $\%$ of fruit infestation.

ubicados a $120 \mathrm{~cm}$ de altura, con el porcentaje de infestación en frutos (100\%) solo se pudo explicar con un modelo cuadrático.

Las trampas de cartón corrugado permitieron generar información de la dinámica poblacional de $P$. viburni en granado, la que no se encontraba disponible en el cultivo, posiblemente debido a la menor importancia de pseudocóccidos en otros lugares del mundo, donde hay otras plagas de mayor importancia que afectan al granado (Mohsenia, 2009; Özturk and Ulusoy, 2009; Balikai et al., 2011; Day et al., 2011), y porque en Chile el granado es un cultivo que se ha expandido recientemente, con pocos estudios más acabados de su condición fitosanitaria (Curkovic et al., 2015). Las observaciones relacionadas a la dinámica poblacional concuerdan con las realizadas en otros frutales de hoja caduca en Chile central: tres generaciones, una en primavera, otra en verano y la última en otoño; una primera generación que demora más tiempo que la segunda (Oyarzún, 2004), y una baja abundancia de individuos expuestos en invierno (Núñez, 2007; Mudavanhu, 2009). La emigración de ninfas observada en junio, desde la fruta hacia el tronco, podría deberse a que en esa época ya ha terminado la cosecha, y los frutos remanentes en los árboles presentan un alto índice de partidura, situación propia del tipo de fruto (balaústa) que crece en forma permanente, incluso luego de la fecha de la cosecha comercial, hasta partirse (Franck et al., 2009). En esas condiciones, la cavidad calicinal de los frutos queda expuesta a la luz, razón por la que, además del consecuente deterioro de la fruta, los chanchitos blancos la abandonan y se desplazan a nuevos sitios buscando oscuridad y protección, por lo que se instalan bajo los cartones corrugados colocados en los troncos.

La ausencia de correlación para un modelo lineal, considerando la relación entre los parámetros climáticos ambientales y la variación de la abundancia de $P$. viburni, podría ser explicado por los hábitos crípticos que tienen estos insectos, que permanecen en refugios pequeños con condiciones microclimáticas más estables y más independientes del medio externo. A pesar de que las variables climáticas no ayudaron a explicar esta fluctuación a lo 
largo del año en el cultivo de granado, las bajas temperaturas y la precipitación parecen tener un efecto en la población en invierno (Fig. 4), situación que Koplow (2004) y Mudavanhu (2009) mencionan en sus trabajos, atribuyéndola a la mortalidad causada por esas condiciones ambientales. Así, la diferencia en los niveles de infestación inicial de $P$. viburni registrados en las dos temporadas en los frutos se condicen con las poblaciones supuestamente sobrevivientes en los respectivos inviernos. Así, en julio de 2012 hubo un déficit en las precipitaciones, y las temperaturas mínimas en general estuvieron por sobre $-2^{\circ} \mathrm{C}$ en la localidad de estudio, mientras que en el año anterior las mínimas alcanzaron valores inferiores de hasta $-^{\circ} \mathrm{C}$ en la misma época (Santibáñez, 2012); esas condiciones de 2012 pueden haber contribuido a que no haya existido una alta mortalidad de individuos de $P$. viburni, y a que se haya presentado un muy alto nivel de infestación ( $80 \%$ árboles) desde inicio de la temporada (Fig. 5). Las características físicas del fruto no explican la alta infestación de chanchito blanco, que alcanzó sobre el $75 \%$ en algunas épocas. Ello sugiere que la infestación es independiente del tamaño y forma del fruto, en el cual, gracias a la presencia de la cavidad calicinal, $P$. viburni encuentra un excelente refugio. Finalmente, los resultados permiten concluir que el seguimiento con trampas de cartón corrugado son una herramienta útil para definir las poblaciones de P. viburni en huertos de granado, y que, junto con el seguimiento en frutos, permitirán optimizar la oportunidad de aplicación de agroquímicos contra esta plaga, para reducir su incidencia en post-cosecha, pero ello deberá incluir estudios de los plaguicidas y métodos de aplicación que ofrezcan períodos de protección apropiados contra P. viburni.

\section{CONCLUSIONES}

El cartón corrugado resultó ser el método más efectivo para el seguimiento de $P$. viburni en granados, especialmente al colocarlo en el tronco a $120 \mathrm{~cm}$ de altura. El muestreo de frutos es complementario, mientras que el de follaje y el uso de cintas doble adhesivas no sirven para este propósito.

P. viburni mostró la ocurrencia de tres generaciones en granado, pero con una prolongada presencia en los frutos (casi 6 meses), dada la tardía cosecha (mayo). Los parámetros climáticos no contribuyen a explicar la variación de la abundancia de $P$. viburni registrada en los cartones a lo largo del tiempo.

No hubo una correlación de las características físicas del fruto con el nivel de infestación de chanchito blanco. La infestación a cosecha fue alta, a pesar de un intenso manejo con agroquímicos en el campo muestreado en ambas temporadas, lo que sugiere la necesidad de una revisión de la estrategia usada respecto de la oportunidad de aplicación, y de los plaguicidas y métodos de aplicación usados.

Estos resultados servirán para definir mejor la oportunidad de aplicación de agroquímicos para el control de P. viburni en granados.

\section{LITERATURA CITADA}

Balikai, R.A., Y.K. Kotikal, and P.M. Prasanna. 2011. Status of pomegranate pests and their management strategies in India. Acta Horticulturae 890:569-583.

Carpio, C. 2013. Bases para el manejo integrado del chanchito blanco (Pseudococcus viburni) en granado (Punica granatum): evaluación de métodos de seguimiento y control. Tesis para obtener el grado de Magister en Ciencias Agropecuarias. Universidad de Chile. Santiago, Chile.

Conover, W.J. 1999. Practical non-parametric statistics. $3^{\text {rd }}$ ed. John Wiley \& Sons, New York, USA.

Curkovic T., y C. Ballesteros. 2011. Manejo integrado de plagas clave del olivo en Copiapó y Huasco. p. 117-144. En Fichet, T., Razeto, B., Curkovic, T. (eds.). El Olivo: Estudio Agronómico en la Región de Atacama. Universidad de Chile, Santiago, Chile.

Curkovic, T., C. Ballesteros, y C. Carpio. 2015. Manejo integrado de plagas del granado. p. 159-231 En Henríquez, J.L. y N. Franck (eds.). Directrices para el Cultivo del Granado en Chile. Universidad de Chile, Santiago, Chile.

Day, K.R., and E.D. Wilkins. 2011. Commercial pomegranate (Punica granatum L.) production in California. Acta Horticulturae 890:275-286.

Franck, N., D. Zamorano, F. Alfaro, A. Peña-Neira, C. Sáenz, y A. Cáceres. 2015. Características de la especie. p. 27-62. En Henríquez, J.L. y N. Franck (eds.). Directrices para el Cultivo del Granado en Chile. Universidad de Chile, Santiago, Chile.

Franck, N., D. Zamorano, F. Alfaro, A. Peña-Neira, C. Sáenz, y A. Cáceres. 2009. Producción y manejo de plantaciones de granado en Chile, Israel y Argentina. p 28-42. En Castillo, C. e I. Quiroz (eds.). Granados, perspectivas y oportunidades de un negocio emergente. Ârea de Agroindustria. Fundación Chile, Santiago, Chile. 
Geiger, C., and K. Daane. 2001.Seasonal movement and distribution of the grape mealybug (Homoptera: Pseudococcidae): developing a sampling program for San Joaquin valley vineyards. Journal of Economic Entomology 94:291-301.

González, R., T. Curkovic, y G. Barria. 1996. Evaluación de eficacia de insecticidas sobre chanchitos blancos en ciruelos y uva de mesa. Revista Frutícola 17(2):45-57.

Henríquez, J.L. 2015. Antecedentes generales, p. 13-26. En Henríquez, J.L. y N. Franck (eds.). Directrices para el cultivo del granado en Chile. Universidad de Chile, Santiago, Chile.

Koplow, C. 2004. Monitoreo y control físico de chanchitos blancos (Pseudococcus viburni) (Signoret) en vid, antecedentes para el manejo integrado. 157 p. Tesis de Magíster. Facultad de Agronomía e Ingeniería Forestal, Pontificia Universidad Católica de Chile, Santiago, Chile.

Mohsenia, A. 2009. The situation of pomegranate orchards in Iran. Acta Horticulturae 818:35-42

Mudavanhu, P. 2009. An investigation into the integrated pest management of the obscure mealybug, Pseudococcus viburni (Signoret) (Hemiptera: Pseudococcidae), in pome fruit orchards in the Western Cape Province, South Africa. 110 p. Master Thesis, College of Agriculture, University of Stellenbosch, South Africa.

Mudavanhu, P., P. Addison, and L. Pringle. 2011. Monitoring and action threshold determination for the obscure mealybug Pseudococcus viburni (Signoret) (Hemiptera: Pseudococcidae) using pheromone baited traps. Crop Protection 30:919-924.

Nuñez, J. 2007. Evaluación de sistemas de monitoreo para chanchitos blancos (Pseudococcus sp.) en vid de mesa. 89 p. Tesis de Magíster. Facultad de Agronomía e Ingeniería Forestal, Pontificia Universidad Católica de Chile, Santiago, Chile.
Oyarzún, M. 2004. Taxonomía y observaciones biológicas del chanchito blanco de los frutales, Pseudococcus viburni (Signoret). (Hemiptera: Pseudococcidae). 85 p. Memoria de Ing. Agrónomo. Facultad de Ciencias Agronómicas, Universidad de Chile, Santiago, Chile,

Ozturk, N., and M.R. Ulusoy. 2009. Pests and natural enemies determined in pomegranate orchards in Turkey. Acta Horticulturae 818:277-284.

Ripa, R., y F. Rodríguez. 1999. Plagas de cítricos, sus enemigos naturales y manejo. $151 \mathrm{p}$. Colección de Libros $\mathrm{N}^{\circ} 3$. Instituto de Investigaciones Agropecuarias, Santiago, Chile.

Santibáñez, P. 2012. Boletín Agroclimático Regional: Región Metropolitana-Período Julio. 14 p. Sistema Agroclimático FDF-INIADMC, Santiago, Chile.

Steel, R., y J. Torrie. 1985. Análisis de la varianza II: clasificaciones múltiples. p. 188-230. En Bioestadística: principios y procedimientos. McGraw-Hill Latinoamericana, Bogotá, Colombia.

Vreysen, M.J.B. 2005. Monitoring sterile and wild insects in area-wide integrated pest management programmes. p. 326-351. In Dyck, V.A., J. Hendrichs and A.S. Robinson (eds.). Sterile Insect: Principles and Practice in Area - Wide Integrated Pest Management. Springer, Dordrecht, The Netherlands.

Waterworth, R., R. Redak, and J.G. Millar. 2011. Pheromone-baited traps for assessment of seasonal activity and population densities of mealybugs species (Hemiptera: Pseudococcidae) in nurseries producing ornamental plants. Journal of Economic Entomology 104:555-565. 